\title{
The association between XRCC3 rs1799794 polymorphism and cancer risk: a meta-analysis of 34 case-control studies
}

Weiqing Liu', Shumin Ma², Lei Liang², Zhiyong Kou ${ }^{2}$ Hongbin Zhang ${ }^{2}$ and Jun Yang ${ }^{2 *}$ (D)

\begin{abstract}
Background: Studies on the XRCC3 rs 1799794 polymorphism show that this polymorphism is involved in a variety of cancers, but its specific relationships or effects are not consistent. The purpose of this meta-analysis was to investigate the association between rs 1799794 polymorphism and susceptibility to cancer.

Methods: PubMed, Embase, the Cochrane Library, Web of Science, and Scopus were searched for eligible studies through June 11, 2019. All analyses were performed with Stata 14.0. Subgroup analyses were performed by cancer type, ethnicity, source of control, and detection method. A total of 37 studies with 23,537 cases and 30,649 controls were included in this meta-analysis.

Results: XRCC3 rs 1799794 increased cancer risk in the dominant model and heterozygous model (GG + AG vs. AA: odds ratio $[O R]=1.04,95 \%$ confidence interval $[\mathrm{Cl}]=1.00-1.08, \mathrm{P}=0.051 ; \mathrm{AG}$ vs. $\mathrm{AA}: \mathrm{OR}=1.05,95 \% \mathrm{Cl}=1.00-1.01$, $P=0.015)$. The existence of rs 1799794 increased the risk of breast cancer and thyroid cancer, but reduced the risk of ovarian cancer. In addition, rs 1799794 increased the risk of cancer in the Caucasian population.

Conclusion: This meta-analysis confirms that XRCC3 rs1799794 is related to cancer risk, especially increased risk for breast cancer and thyroid cancer and reduced risk for ovarian cancer. However, well-designed large-scale studies are required to further evaluate the results.
\end{abstract}

Keywords: Rs1799794, Polymorphism, Cancer, Risk, Meta-analysis

\section{Background}

Cancer is the leading cause of death worldwide, and the number of patients with cancer is increasing [1]. The occurrence of cancer is related to many factors, including environmental, lifestyle, genetic and other factors. Among them, gene mutation is a kind of genetic factor, which has a great influence on cancer risk [2]. The mutation in BRCA1 and BRCA2 is related to the increase risk

*Correspondence: yangjun6@kmmu.edu.cn

${ }^{2}$ Department of Oncology, the First Affiliated Hospital of Kunming

Medical University, Yunnan Province, No. 295 Xichang Road,

Kunming 650032, People's Republic of China

Full list of author information is available at the end of the article of breast cancer [3]. XPF rs2276466 polymorphism is related to neurogenic cancer [4].

X-ray repair cross-complementing group 3 (XRCC3), functions in the homologous recombination (HR) repair of DNA crosslinks [5] and double-strand breaks [6]. Based on the function of XRCC3, XRCC3 gene mutations are related to the occurrence and development of many diseases. For example, XRCC3 241Thr/Met genotype promotes left ventricular hypertrophy by inhibiting DNA damage repair [7]. Mutations in the XRCC3 gene affect mitochondrial DNA integrity [8]. XRCC3 rs861539 polymorphism is associated with poor prognosis of breast cancer patients [9]. The mutation sites that have been studied more about the relationship between 
XRCC3 gene and cancer are rs861539, rs1799794 and rs1799796 [10]. However, results remain fairly conflicting rather than conclusive. A number of meta-analyses have investigated the relationship between rs861539 and susceptibility to various cancers [11-33]. However, there have been few meta-studies on rs1799794 and susceptibility to cancer $[28,30,31,33,34]$. Therefore, we conducted this meta-analysis to analyze the relationship between rs1799794 and susceptibility to cancer on the basis of more data.

\section{Methods}

\section{Search strategies}

We comprehensively searched five databases (PubMed, Embase, the Cochrane Library, Web of Science, and Scopus) for research published as of June 11, 2019, using relevant $\mathrm{MeSH}$ terms and entry terms. The keywords of XRCC3 included X-ray repair cross complementing 3, rs1799794, 4541A/G, XRCC3. The MeSH term and entry terms of polymorphism were genetic polymorphism [MeSH terms]; polymorphisms, genetic; genetic polymorphisms; genetic polymorphism; polymorphism (genetics); polymorphisms (genetics); polymorphism, single nucleotide; nucleotide polymorphism, single; nucleotide polymorphisms, single; polymorphisms, single nucleotide; single nucleotide polymorphisms; polymorphisms; polymorphism; variant; mutation; single nucleotide polymorphism; SNP. The MeSH term and entry terms of cancer were neoplasm [MeSH terms], neoplasms, neoplasia, neoplasias, neoplasm, tumors, tumor, cancer, cancers, carcinoma, carcinogenesis, tumour. Furthermore, we refined the search results of related studies by looking at the list of references included in each article.

\section{Selection criteria}

Relevant studies were included in accordance with the inclusion criteria and exclusion criteria, which were similar to those described in the previous study (PMID: 30867406). Original case-control study focused on the relationship between rs1799794 and cancer risk with the frequency of XRCC3 rs1799794 mutant genotypes were included. While conference abstracts or reports, reviews or meta-analyses, republished articles, and studies with insufficient data were excluded.

\section{Data extraction and quality assessment}

The following data from each selected article were collected: the surname of the first author, the publication year, country, ethnicity, cancer types, and methods of genotyping XRCC3 rs1799794 polymorphism. The quality of eligible case-control studies was estimated using the Newcastle-Ottawa Scale [35].

\section{Statistical analysis}

The relationship between XRCC3 rs1799794 polymorphisms and cancer risk were evaluated using odds ratios (ORs) and 95\% confidence intervals (CI) under five genetic models ( $G$ vs. A, GG vs. AA, GG+GA vs. AA, GG vs. GA + AA, GA vs. AA).as previous study. If $\mathrm{P}<0.05$ or the $95 \%$ CI did not include 1 , the result was considered statistically significant. Cochran's $Q$ with chi-square (with $\mathrm{P}_{\mathrm{Q}}$ ) and the Higgins $\mathrm{I}^{2}$ test were used to determine heterogeneity in between-study variability. When $\mathrm{P}_{\mathrm{Q}}<0.1$ or $\mathrm{I}^{2}>25 \%$ indicated significant heterogeneity [36-38], we analyzed the data using a random effects model [39]. If the opposite held, a fixed effects model was chosen. We also performed subgroup analyses and a sensitivity analysis to explore sources of heterogeneity. Subgroup analyses stratified studies by cancer type (ovarian cancer, acute lymphoblastic leukemia, breast cancer, thyroid cancer, bladder cancer, lung cancer, other), ethnicity (Arabian, Asian, Caucasian, mixed), sample size $(<100,>100)$, the publication year $(\leq 2010,>2010)$, detection method (PCR-RFLP, sequencing, TaqMan, PCR, ND, other), and source of control (HB, $\mathrm{PB}$, mixed, nested). We assessed publication bias using funnel plots and Egger's test $(\mathrm{P}<0.05)$. Statistical calculations were performed with Stata 14.0.

\section{Results}

\section{Literature search and study characteristics}

Finally, 3,467 potentially relevant published works were identified (997 in PubMed, 27 in the Cochrane library, 855 in Embase, 696 in Scopus, and 889 in Web of Science). Of these, duplicates (1959) and works not related to cancer and rs1799794 polymorphism (1451) were excluded. Then 23 of these studies were excluded after reviewing full texts. The remaining 37 works (43 studies) were included in this meta-analysis [10, 40-75]. Because two studies in Auranen et al. [10] were duplicated in Quaye et al. [62], we only extracted data from these studies from Auranen et al. [10] to avoid duplication; thus, one article included four studies [66], and three articles included two studies each $[10,68,70]$. The flow chart of the literature selection process is shown in Fig. 1.

There were a total of 23,537 cases and 30,649 controls in these 37 works, and 3 were conducted among Arabians [40, 48, 55], 14 among Asians [41, 42, 45-47, 49, 50, 53, $54,56,58,59,66,67]$, and 24 among Caucasians [10, 43, $44,51,52,57,60-62,64,66,69-75]$; 2 were conducted among mixed populations $[63,65]$. In addition, in terms of cancer type, ovarian cancer $(\mathrm{n}=4)[10,40,62]$, acute lymphoblastic leukemia $(\mathrm{n}=3)[41,52,57]$, breast cancer $(\mathrm{n}=13)[44,48,49,55,61,66,68,72,74]$, thyroid cancer $(n=4)[42,46,47,67]$, bladder cancer $(n=4)[45,63$, 


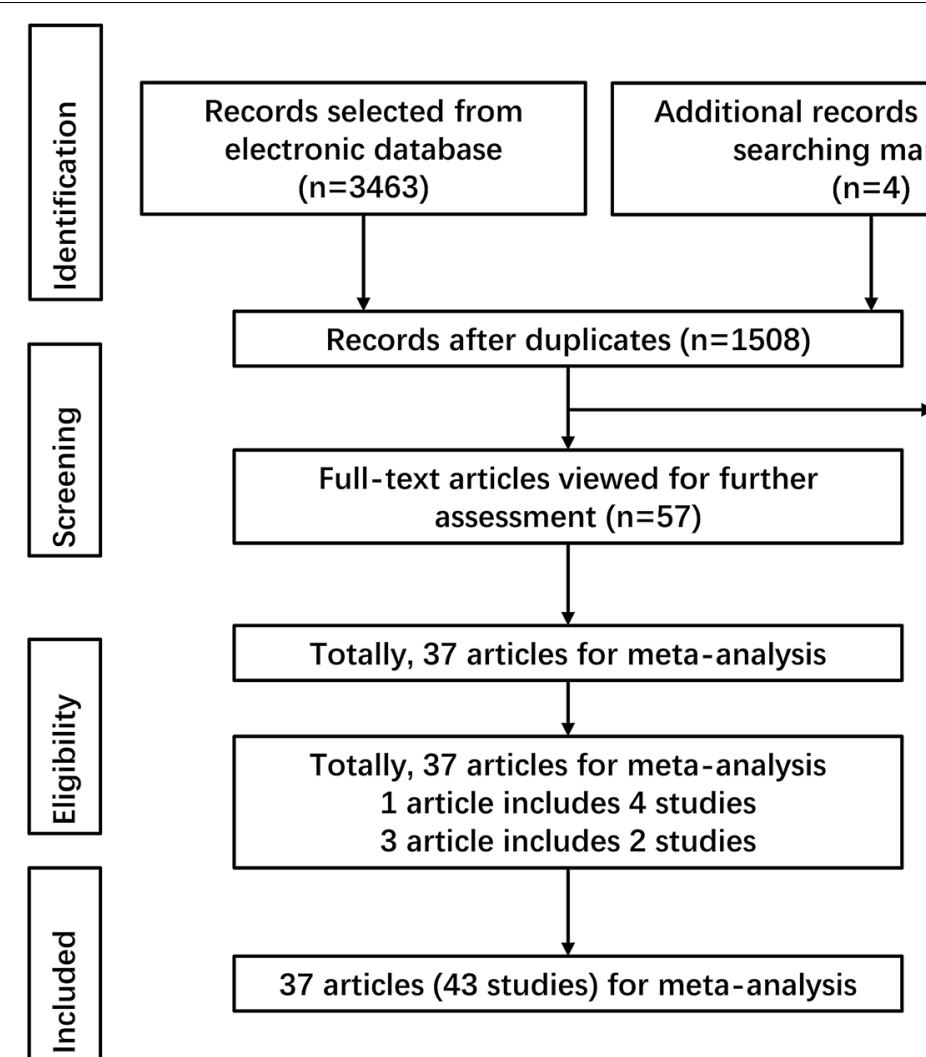

Fig. 1 Flow chart of study selection

$65,69]$, lung cancer $(n=3)[53,59,71]$, and other cancer (hepatocellular cancer, leiomyoma, nasopharyngeal carcinoma, osteosarcoma, oral cancer, glioma, head and neck cancer, myeloma, endometrial cancer, colorectal adenoma, melanoma skin cancer) $[43,50,51,54,56,58$, $60,64,70,73,75]$ were studied. The basic information of each study is presented in Table 1 . And we took sensitivity analysis for studies that do not conform to HWE.

\section{Meta-analysis and subgroup analyses}

The value of $\mathrm{I}^{2}$ in the five genetic models was greater than $25 \%$, and $\mathrm{P}_{\mathrm{Q}}<0.10$, so pooled ORs for the five genetic models were calculated with a random effects model. There was no obvious correlation between rs1799794 and cancer risk $\left(\mathrm{P}_{Z}>0.05\right.$; Table 2$)$.

Subgroup analyses were then performed based on cancer type, ethnicity, detection method, the publication year, source of control, and sample size to investigate sources of heterogeneity (Table 3 ). In the subgroup analysis based on cancer type, a significantly increased risk for thyroid cancer was observed in the five models ( $G$ vs. A: $\mathrm{OR}=1.27,95 \% \mathrm{CI}=1.01-1.61, \mathrm{I}^{2}=71.2 \% ; \mathrm{GG}+\mathrm{AG}$ vs. $\mathrm{AA}: \mathrm{OR}=1.36,95 \% \mathrm{CI}=1.15-1.61, \mathrm{I}^{2}=55.4 \%$; GG vs. $\mathrm{AA}+\mathrm{AG}$ : $\mathrm{OR}=1.38,95 \% \mathrm{CI}=1.09-1.75, \mathrm{I}^{2}=29.8 \%$;
GG vs. $A A: O R=1.50,95 \% C I=1.17-1.93, \mathrm{I}^{2}=45.7 \%$; $\mathrm{AG}$ vs. $\mathrm{AA}: \mathrm{OR}=1.27,95 \% \mathrm{CI}=1.05-1.53, \mathrm{I}^{2}=33.2 \%$ ), a significantly increased risk for breast cancer was found in the heterozygous model $(\mathrm{OR}=1.08,95 \% \mathrm{CI}=1.02-$ $\left.1.13, \mathrm{I}^{2}=42.3 \%\right)$, and a decreased risk for ovarian cancer was found in the recessive model and homozygous model (GG vs. AA + AG: $\mathrm{OR}=0.69,95 \% \mathrm{CI}=0.51-0.93$, $\mathrm{I}^{2}=0.0 \% ; \mathrm{GG}$ vs. AA: $\mathrm{OR}=0.71,95 \% \mathrm{CI}=0.53-0.96$, $\left.\mathrm{I}^{2}=0.0 \%\right)$.

In the subgroup analysis based on ethnicity, rs1799794 was associated with increased cancer risk in the Caucasian population according to the heterozygous model (AG vs. $\mathrm{AA}: \mathrm{OR}=1.05,95 \% \mathrm{CI}=1.01-1.10, \mathrm{I}^{2}=0.0 \%$ ). In the subgroup analysis based on source of control, we found a significantly increased risk for PB (population based) in the dominant model and heterozygous model $(\mathrm{GG}+\mathrm{AG}$ vs. AA: $\mathrm{OR}=1.06,95 \% \mathrm{CI}=1.01-1.12$, $\mathrm{I}^{2}=0.0 \% ;$ AG vs. AA: OR $=1.09,95 \% \mathrm{CI}=1.03-1.15$, $\left.\mathrm{I}^{2}=0.0 \%\right)$. In the subgroup analysis based on detection method, sequencing was associated with a significantly increased cancer risk in the allele model, dominant model, and heterozygous model ( $G$ vs. A: OR $=2.60$, 95\% $\mathrm{CI}=1.37-4.94, \mathrm{I}^{2}=0.0 \%$; $\mathrm{GG}+\mathrm{AG}$ vs. $\mathrm{AA}: \mathrm{OR}=4.00$, $95 \% \mathrm{CI}=1.82-8.80, \mathrm{I}^{2}=0.0 \%$; AG vs. AA: OR $=4.00,95 \%$ 


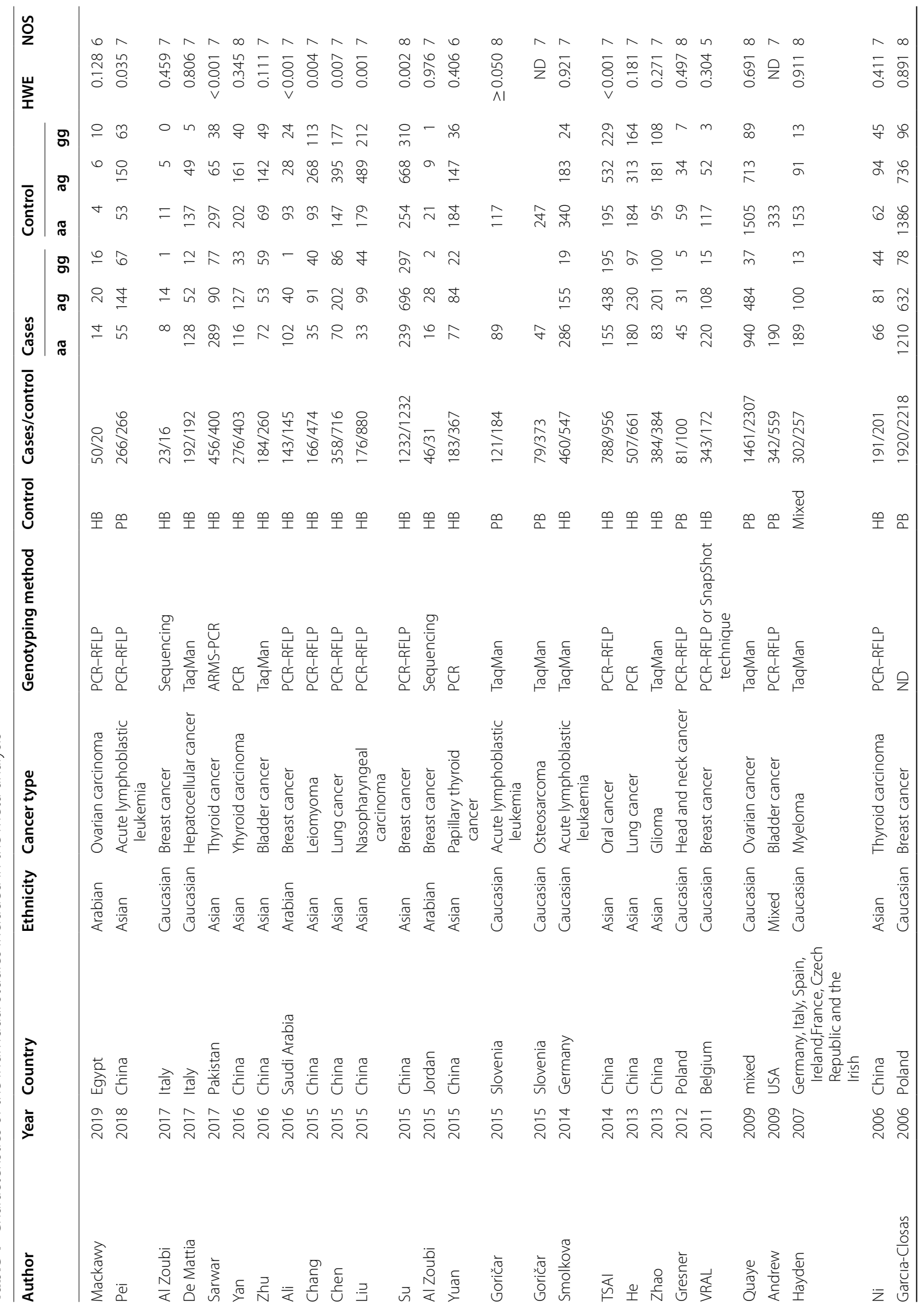




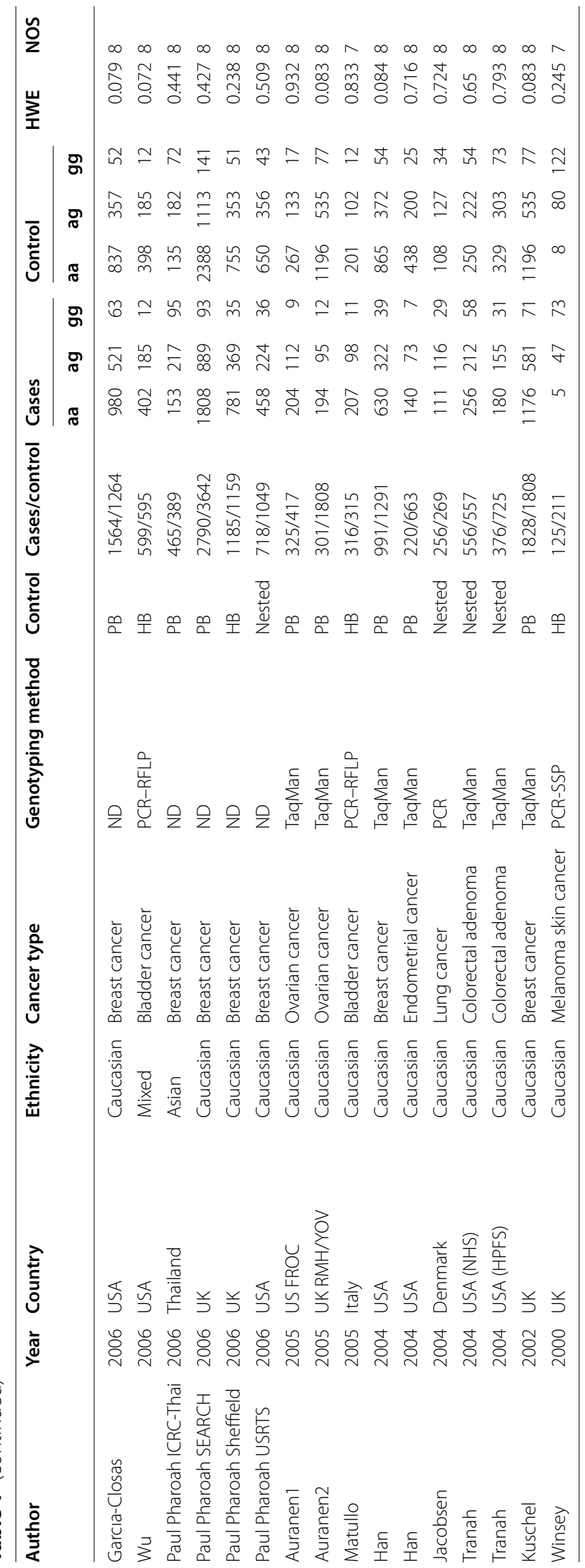


Table 2 The results of the meta-analyses under different genetic models for all studies

\begin{tabular}{llllll}
\hline Genetic model & Number & $\mathbf{I}^{\mathbf{2}}(\mathbf{\%})$ & $\mathbf{P}_{\mathbf{H}}$ & $\mathbf{O R}(\mathbf{9 5} \% \mathbf{C I})$ & $\mathbf{P}_{\mathbf{Z}}$ \\
\hline G VS A & 40 & 47.50 & 0.001 & $1.02(0.98-1.07)$ & 0.377 \\
GG VS AA & 40 & 30.20 & 0.039 & $0.98(0.89-1.08)$ & 0.713 \\
GG + GA VS AA & 43 & 40.0 & 0.004 & $1.04(0.98-1.09)$ & 0.207 \\
GG VS GA + AA & 40 & 34.10 & 0.02 & $0.98(0.90-1.07)$ & 0.696 \\
GA VS AA & 40 & 39.40 & 0.006 & $1.04(0.99-1.11)$ & 0.134 \\
\hline
\end{tabular}

$\left.\mathrm{CI}=1.79-8.94, \mathrm{I}^{2}=0.0 \%\right)$. In the subgroup analysis based on sample size, AG carriers were 2.82 times more likely to develop cancer than AA carriers $(95 \% \mathrm{CI}=1.42-5.57$, $\left.\mathrm{P}_{\mathrm{Z}}=0.003\right)$. In the subgroup analysis based on the publication year, studies published before 2010 showed that AG carriers were 1.05 times more likely to develop cancer than AA carriers $\left(95 \% \mathrm{CI}=1.00-1.10, \mathrm{P}_{\mathrm{Z}}=0.047\right)$.

\section{Publication bias}

The shape of the funnel plots (Fig. 2) and Egger's test (allele: $\mathrm{P}=0.108$, dominant: $\mathrm{P}=0.177$, recessive: $\mathrm{P}=0.240$, homozygous: $\mathrm{P}=0.132$, heterozygous: $\mathrm{P}=0.177$ ) showed no publication bias.

\section{Sensitivity analysis}

Eight studies $[41,42,48-50,53,54,56]$ had $\mathrm{P}_{\mathrm{HWE}}<0.05$, but for two studies $[51,63] \mathrm{P}_{\text {HWE }}$ was not available. We compared the combined results before and after excluding these 10 studies and there were slight changes in the results. When the subgroup analysis was performed according to cancer type, there were no significant associations between rs1799794 polymorphism and increased risk for thyroid cancer in the recessive model, homozygous model, or heterozygous model (GG vs. $\mathrm{AA}+\mathrm{AG}: \mathrm{OR}=1.16,95 \% \mathrm{CI}=0.87-1.55, \mathrm{I}^{2}=0.0 \%$; GG vs. $\mathrm{AA}: \mathrm{OR}=1.24,95 \% \mathrm{CI}=0.90-1.69, \mathrm{I}^{2}=0.0 \%$; $\mathrm{AG}$ vs. $\left.\mathrm{AA}: \mathrm{OR}=1.22,95 \% \mathrm{CI}=0.98-1.51, \mathrm{I}^{2}=49.4 \%\right)$, and rs3116496 was related to a decreased risk for lung cancer in the five models (A vs. G: $\mathrm{OR}=0.80,95 \% \mathrm{CI}=0.70$ $0.92, \mathrm{I}^{2}=18.1 \% ; \mathrm{GG}+\mathrm{AG}$ vs. $\mathrm{AA}: \mathrm{OR}=0.76,95 \%$ $\mathrm{CI}=0.62-0.93, \mathrm{I}^{2}=4.9 \%$; $\mathrm{GG}$ vs. $\mathrm{AA}+\mathrm{AG}: \mathrm{OR}=0.75$, $95 \% \mathrm{CI}=0.59-0.96, \mathrm{I}^{2}=0.0 \%$; GG vs. $\mathrm{AA}: \mathrm{OR}=0.65$, $95 \% \mathrm{CI}=0.49-0.87, \mathrm{I}^{2}=0.0 \%$; AG vs. AA: OR $=0.80,95 \%$ $\left.\mathrm{CI}=0.64-0.99, \mathrm{I}^{2}=0.0 \%\right)$; no changes were observed for the other cancers. No significant changes were found in the subgroup analyses by ethnicity and source of control.

\section{Discussion}

Our study shows that XRCC3 rs1799794 is irrelevant to cancer risk. In addition, the risk for thyroid cancer and breast cancer increase significantly in patients with rs1799794, and Caucasian populations are more likely to develop these cancers while having a decreased risk for ovarian cancer. We excluded articles that did not conform to HWE and reanalyzed the data. Compared to the previous results, rs3116496 was related to a decreased risk for lung cancer in the five models, although the other results were not much changed (data not shown).

Moderate heterogeneity was found in this meta-analysis. First, we used random models when significant heterogeneity. Second, we performed subgroup analyses to explore sources of heterogeneity. As shown in Table 3, in the subgroup analysis based on ethnicity, heterogeneity increased in Arabian/Asian populations but was $0 \%$ in Caucasian populations, which suggests that ethnicity may be a factor in heterogeneity. Furthermore, we analyzed studies stratified by cancer type, detection method, source of control, and sample size. Ethnicity, cancer type, source of control, and sample size may be the source of inter-research heterogeneity. In addition, a sensitivity analysis suggested that the current findings were reliable.

To date, five meta-analyses of the impact of rs1799794 on cancer risk have been performed [28, 30, 31, 33, 34] on rs1799794 and susceptibility to pan-cancer [28], breast cancer [30,34], bladder cancer [33], and ovarian cancer [31]. To the best of our knowledge, ours is currently the most comprehensive meta-analysis of correlations between rs1799794 polymorphisms and cancer. There are many differences between the results of this study and previous studies. According to Qiu et al's research on rs1799794 and susceptibility to breast cancer, which included four studies in three papers, rs1799794 was associated with a statistically significant increase in cancer risk in the dominant model $(\mathrm{GG}+\mathrm{AG}$ vs. AA: $\left.\mathrm{OR}=1.09,95 \% \mathrm{CI}=1.01-1.17, \mathrm{P}_{\mathrm{H}}=0.15\right)$, whereas our results showed an increased risk for breast cancer in AG carriers, different from the protective effect found previously [48]. In addition, our study found that the $G$ allele might be a dominant gene and found an increased risk for thyroid cancer.

Our study included a large number of samples and conducted a stratified analysis, which played an important role in the reliability of the research results. At the same time, there are problems that cannot be ignored: the presence of heterogeneity that may due to ethnicity, source of control, status, or cancer type; the lack of relevant data published in other languages and evaluation of the interaction between cancer-related factors.

\section{Conclusion}

In conclusion, this meta-analysis found no association between XRCC3 rs1799794 and cancer risk, but XRCC3 rs1799794 was associated with breast cancer and thyroid cancer as well as with Caucasian 
Table 3 Results of meta-analysis for polymorphisms in different subgroups and cancer susceptibility

\begin{tabular}{|c|c|c|c|c|c|c|}
\hline Comparison & Subgroup & Number & $1^{2}$ & $\mathrm{P}_{\mathrm{H}}$ & $\mathrm{P}_{\mathrm{Z}}$ & OR $(95 \% \mathrm{Cl})$ \\
\hline \multirow[t]{31}{*}{ GVS A } & Ethnicity & & & & & \\
\hline & Arabian & 3 & $84.9 \%$ & 0.001 & 0.752 & $0.86(0.33-2.23)$ \\
\hline & Asian & 14 & $64.8 \%$ & $P<0.001$ & 0.255 & $1.05(0.96-1.15)$ \\
\hline & Caucasian & 22 & $0.0 \%$ & 0.661 & 0.502 & $1.01(0.98-1.05)$ \\
\hline & Mixed & 1 & NA & NA & 0.940 & $0.99(0.80-1.23)$ \\
\hline & Cancer type & & & & & \\
\hline & Ovarian cancer & 4 & $0.0 \%$ & 0.547 & 0.848 & $0.99(0.90-1.09)$ \\
\hline & Acute lymphoblastic leukemia & 2 & $0.0 \%$ & 0.887 & 0.979 & $1.00(0.85-1.18)$ \\
\hline & Breast cancer & 13 & $58.6 \%$ & 0.004 & 0.494 & $1.03(0.95-1.10)$ \\
\hline & Thyroid cancer & 4 & $71.2 \%$ & 0.015 & 0.043 & $1.27(1.01-1.61)$ \\
\hline & Bladder cancer & 3 & $0.0 \%$ & 0.921 & 0.815 & $0.98(0.85-1.13)$ \\
\hline & lung cancer & 3 & $60.1 \%$ & 0.082 & 0.166 & $0.88(0.74-1.05)$ \\
\hline & Others & 11 & $0.0 \%$ & 0.902 & 0.822 & $1.01(0.91-1.08)$ \\
\hline & Method & & & & & \\
\hline & PCR-RFLP & 12 & $22.3 \%$ & 0.225 & 0.657 & $0.99(0.93-1.05)$ \\
\hline & Sequencing & 2 & $0.0 \%$ & 0.828 & 0.004 & $2.60(1.37-4.94)$ \\
\hline & TaqMan & 13 & $0.0 \%$ & 0.886 & 0.475 & $1.02(0.97-1.07)$ \\
\hline & PCR & 4 & $82.4 \%$ & 0.001 & 0.913 & $1.02(0.78-1.33)$ \\
\hline & ND & 6 & $14.6 \%$ & 0.321 & 0.663 & $1.01(0.96-1.06)$ \\
\hline & Others & 3 & $68.3 \%$ & 0.043 & 0.089 & $1.32(0.96-1.82)$ \\
\hline & Source of control & & & & & \\
\hline & $\mathrm{HB}$ & 23 & $66.0 \%$ & $P<0.001$ & 0.445 & $1.03(0.95-1.13)$ \\
\hline & PB & 12 & $0.0 \%$ & 0.892 & 0.135 & $1.03(0.99-1.08)$ \\
\hline & Mixed & 1 & NA & NA & 0.442 & $0.89(0.67-1.19)$ \\
\hline & Nested & 4 & $0.0 \%$ & 0.874 & 0.294 & $0.95(0.86-1.05)$ \\
\hline & Sample size & & & & & \\
\hline & $<100$ & 3 & $77.1 \%$ & 0.013 & 0.419 & $1.54(0.54-4.43)$ \\
\hline & $>100$ & 37 & $43.7 \%$ & 0.003 & 0.424 & $1.02(0.98-1.07)$ \\
\hline & Year & & & & & \\
\hline & $\leq 2010$ & 20 & $0.0 \%$ & 0.910 & 0.700 & $1.01(0.97-1.04)$ \\
\hline & $>2010$ & 20 & $69.5 \%$ & 0.000 & 0.272 & $1.06(0.96-1.17)$ \\
\hline \multirow[t]{18}{*}{$\mathrm{GG}+\mathrm{AG}$ VS AA } & Ethnicity & & & & & \\
\hline & Arabian & 3 & $79.8 \%$ & 0.007 & 0.739 & $1.21(0.39-3.76)$ \\
\hline & Asian & 14 & $64.4 \%$ & $P<0.001$ & 0.547 & $1.04(0.91-1.20)$ \\
\hline & Caucasian & 24 & $0.6 \%$ & 0.453 & 0.119 & $1.03(0.99-1.08)$ \\
\hline & Mixed & 2 & $0.0 \%$ & 0.620 & 0.765 & $1.03(0.85-1.24)$ \\
\hline & Cancer type & & & & & \\
\hline & Ovarian cancer & 4 & $0.0 \%$ & 0.887 & 0.439 & $1.05(0.93-1.17)$ \\
\hline & Acute lymphoblastic leukemia & 3 & $24.4 \%$ & 0.267 & 0.397 & $0.90(0.75-1.12)$ \\
\hline & Breast cancer & 13 & $47.0 \%$ & 0.031 & 0.037 & $1.06(0.98-1.15)$ \\
\hline & Thyroid cancer & 4 & $55.4 \%$ & 0.081 & 0.033 & $1.36(1.15-1.61)$ \\
\hline & Bladder cancer & 4 & $59.1 \%$ & 0.062 & 0.370 & $0.89(0.70-1.14)$ \\
\hline & Lung cancer & 3 & $51.2 \%$ & 0.129 & 0.207 & $0.85(0.66-1.09)$ \\
\hline & Others & 12 & $0.0 \%$ & 0.910 & 0.597 & $1.03(0.93-1.13)$ \\
\hline & Method & & & & & \\
\hline & PCR-RFLP & 13 & $0.0 \%$ & 0.965 & 0.840 & $1.01(0.92-1.11)$ \\
\hline & Sequencing & 2 & $0.0 \%$ & 0.956 & 0.001 & $4.00(1.82-8.80)$ \\
\hline & TaqMan & 15 & $29.2 \%$ & 0.137 & 0.269 & $1.04(0.97-1.10)$ \\
\hline & PCR & 4 & $81.0 \%$ & 0.001 & 0.862 & $1.03(0.72-1.48)$ \\
\hline
\end{tabular}


Table 3 (continued)

\begin{tabular}{|c|c|c|c|c|c|c|}
\hline Comparison & Subgroup & Number & $1^{2}$ & $P_{H}$ & $\mathrm{P}_{\mathrm{z}}$ & OR $(95 \% \mathrm{Cl})$ \\
\hline & ND & 6 & $28.0 \%$ & 0.225 & 0.360 & $1.03(0.97-1.09)$ \\
\hline & Others & 3 & $16.0 \%$ & 0.304 & 0.051 & $1.45(1.15-1.82)$ \\
\hline & \multicolumn{6}{|l|}{ Source of control } \\
\hline & $\mathrm{HB}$ & 23 & $58.4 \%$ & $P<0.001$ & 0.397 & $1.05(0.94-1.18)$ \\
\hline & PB & 15 & $0.0 \%$ & 0.656 & 0.015 & $1.06(1.01-1.12)$ \\
\hline & Mixed & 1 & NA & NA & 0.461 & $0.88(0.63-1.24)$ \\
\hline & Nested & 4 & $0.0 \%$ & 0.979 & 0.190 & $0.92(0.82-1.04)$ \\
\hline & \multicolumn{6}{|l|}{ Sample size } \\
\hline & $<100$ & 3 & $65.8 \%$ & 0.054 & 0.179 & $2.23(0.69-7.21)$ \\
\hline & $>100$ & 40 & $32.9 \%$ & 0.025 & 0.234 & $1.03(0.98-1.09)$ \\
\hline & \multicolumn{6}{|l|}{ Year } \\
\hline & $\leq 2010$ & 21 & $0.0 \%$ & 0.815 & 0.166 & $1.03(0.99-1.08)$ \\
\hline & $>2010$ & 22 & $62.0 \%$ & 0.000 & 0.322 & $1.07(0.94-1.22)$ \\
\hline \multirow[t]{31}{*}{ GGVS AA+AG } & \multicolumn{6}{|l|}{ Ethnicity } \\
\hline & Arabian & 3 & $73.9 \%$ & 0.022 & 0.218 & $0.28(0.04-2.13)$ \\
\hline & Asian & 14 & $52.7 \%$ & 0.011 & 0.253 & $1.08(0.95-1.23)$ \\
\hline & Caucasian & 22 & $0.0 \%$ & 0.806 & 0.056 & $0.91(0.82-1.00)$ \\
\hline & Mixed & 1 & NA & NA & 0.987 & $0.99(0.44-2.23)$ \\
\hline & \multicolumn{6}{|l|}{ Cancer type } \\
\hline & Ovarian cancer & 4 & $0.0 \%$ & 0.678 & 0.014 & $0.69(0.51-0.93)$ \\
\hline & Acute lymphoblastic leukemia & 2 & $0.0 \%$ & 0.698 & 0.818 & $1.04(0.75-1.45)$ \\
\hline & Breast cancer & 13 & $35.7 \%$ & 0.097 & 0.101 & $0.92(0.83-1.02)$ \\
\hline & Thyroid cancer & 4 & $29.8 \%$ & 0.234 & 0.007 & $1.38(1.09-1.75)$ \\
\hline & Bladder cancer & 3 & $52.3 \%$ & 0.123 & 0.303 & $1.35(0.76-2.37)$ \\
\hline & Lung cancer & 3 & $5.5 \%$ & 0.347 & 0.062 & $0.83(0.69-1.01)$ \\
\hline & Others & 11 & $0.0 \%$ & 0.893 & 0.993 & $1.00(0.88-1.13)$ \\
\hline & \multicolumn{6}{|l|}{ Method } \\
\hline & PCR-RFLP & 12 & $18.3 \%$ & 0.265 & 0.421 & $0.96(0.86-1.06)$ \\
\hline & Sequencing & 2 & $0.0 \%$ & 0.818 & 0.621 & $1.63(0.23-11.46)$ \\
\hline & TaqMan & 13 & $41.3 \%$ & 0.059 & 0.462 & $0.95(0.84-1.08)$ \\
\hline & PCR & 4 & $44.2 \%$ & 0.146 & 0.211 & $0.88(0.71-1.08)$ \\
\hline & ND & 6 & $8.8 \%$ & 0.360 & 0.363 & $0.94(0.81-1.08)$ \\
\hline & Others & 3 & $60.9 \%$ & 0.078 & 0.121 & $1.54(0.89-2.64)$ \\
\hline & \multicolumn{6}{|l|}{ Source of control } \\
\hline & $\mathrm{HB}$ & 23 & $55.0 \%$ & 0.010 & 0.614 & $1.04(0.90-1.20)$ \\
\hline & PB & 12 & $0.0 \%$ & 0.862 & 0.111 & $0.91(0.81-1.02)$ \\
\hline & Mixed & 1 & NA & NA & 0.674 & $0.84(0.38-1.02)$ \\
\hline & Nested & 4 & $0.0 \%$ & 0.536 & 0.967 & $1.00(0.80-1.24)$ \\
\hline & \multicolumn{6}{|l|}{ Sample size } \\
\hline & $<100$ & 3 & $0.0 \%$ & 0.537 & 0.339 & $0.64(0.26-1.59)$ \\
\hline & $>100$ & 37 & $36.9 \%$ & 0.014 & 0.766 & $0.99(0.90-1.07)$ \\
\hline & \multicolumn{6}{|l|}{ Year } \\
\hline & $\leq 2010$ & 20 & $0.0 \%$ & 0.928 & 0.068 & $0.94(0.83-1.01)$ \\
\hline & $>2010$ & 20 & $58.0 \%$ & 0.001 & 0.374 & $1.08(0.92-1.27)$ \\
\hline \multirow[t]{5}{*}{ GG VS AA } & \multicolumn{6}{|l|}{ Ethnicity } \\
\hline & Arabian & 3 & $75.4 \%$ & 0.017 & 0.338 & $0.33(0.04-3.15)$ \\
\hline & Asian & 14 & $47.8 \%$ & 0.024 & 0.279 & $1.08(0.93-1.26)$ \\
\hline & Caucasian & 22 & $0.0 \%$ & 0.812 & 0.083 & $0.91(0.82-1.01)$ \\
\hline & Mixed & 1 & NA & NA & 0.981 & $0.99(0.44-2.23)$ \\
\hline
\end{tabular}


Table 3 (continued)

\begin{tabular}{|c|c|c|c|c|c|c|}
\hline Comparison & Subgroup & Number & $1^{2}$ & $P_{H}$ & $\mathrm{P}_{\mathrm{z}}$ & OR $(95 \% \mathrm{Cl})$ \\
\hline & \multicolumn{6}{|l|}{ Cancer type } \\
\hline & Ovarian cancer & 4 & $0.0 \%$ & 0.705 & 0.028 & $0.71(0.53-0.96)$ \\
\hline & Acute lymphoblastic leukemia & 2 & $0.0 \%$ & 0.836 & 0.961 & $0.99(0.67-1.47)$ \\
\hline & Breast cancer & 13 & $37.7 \%$ & 0.082 & 0.311 & $0.94(0.85-1.05)$ \\
\hline & Thyroid cancer & 4 & $45.7 \%$ & 0.137 & 0.001 & $1.50(1.17-1.93)$ \\
\hline & Bladder cancer & 3 & $0.0 \%$ & 0.860 & 0.773 & $1.06(0.72-1.55)$ \\
\hline & Lung cancer & 3 & $53.1 \%$ & 0.119 & 0.019 & $0.79(0.56-1.11)$ \\
\hline & Others & 11 & $0.0 \%$ & 0.884 & 0.798 & $1.02(0.88-1.19)$ \\
\hline & \multicolumn{6}{|l|}{ Method } \\
\hline & PCR-RFLP & 12 & $10.7 \%$ & 0.340 & 0.591 & $0.96(0.85-1.10)$ \\
\hline & Sequencing & 2 & $0.0 \%$ & 0.837 & 0.264 & $3.09(0.43-22.45)$ \\
\hline & TaqMan & 13 & $0.0 \%$ & 0.701 & 0.297 & $0.93(0.81-1.07)$ \\
\hline & PCR & 4 & $73.8 \%$ & 0.010 & 0.937 & $0.98(0.61-1.58)$ \\
\hline & ND & 6 & $2.7 \%$ & 0.399 & 0.436 & $0.94(0.82-1.09)$ \\
\hline & Others & 3 & $0.0 \%$ & 0.409 & $P<0.001$ & $1.97(1.36-2.87)$ \\
\hline & \multicolumn{6}{|l|}{ Source of control } \\
\hline & $\mathrm{HB}$ & 23 & $52.8 \%$ & 0.002 & 0.628 & $1.04(0.88-1.24)$ \\
\hline & PB & 12 & $0.0 \%$ & 0.911 & 0.185 & $0.92(0.82-1.04)$ \\
\hline & Mixed & 1 & NA & NA & 0.604 & $0.81(0.36-1.80)$ \\
\hline & Nested & 4 & $0.0 \%$ & 0.553 & 0.737 & $0.96(0.76-1.21)$ \\
\hline & \multicolumn{6}{|l|}{ Sample size } \\
\hline & $<100$ & 3 & $18.0 \%$ & 0.295 & 0.796 & $0.87(0.31-2.48)$ \\
\hline & $>100$ & 37 & 32.5 & 0.031 & 0.733 & $0.98(0.89-1.08)$ \\
\hline & \multicolumn{6}{|l|}{ Year } \\
\hline & $\leq 2010$ & 20 & $0.0 \%$ & 0.961 & 0.070 & $0.91(0.82-1.01)$ \\
\hline & $>2010$ & 20 & $55.2 \%$ & 0.002 & 0.356 & $1.06(0.96-1.17)$ \\
\hline \multirow[t]{23}{*}{ AG VS AA } & \multicolumn{6}{|l|}{ Ethnicity } \\
\hline & Arabian & 3 & $54.9 \%$ & 0.109 & 0.174 & $1.76(0.78-3.95)$ \\
\hline & Asian & 14 & $65.7 \%$ & $P<0.001$ & 0.906 & $1.01(0.86-1.18)$ \\
\hline & Caucasian & 22 & $0.0 \%$ & 0.631 & 0.023 & $1.05(1.01-1.10)$ \\
\hline & Mixed & 1 & NA & NA & 0.937 & $0.99(0.77-1.27)$ \\
\hline & \multicolumn{6}{|l|}{ Cancer type } \\
\hline & Ovarian cancer & 4 & $0.0 \%$ & 0.998 & 0.145 & $1.09(0.97-1.22)$ \\
\hline & Acute lymphoblastic leukemia & 2 & $0.0 \%$ & 0.747 & 0.893 & $0.98(0.78-1.24)$ \\
\hline & Breast cancer & 13 & $42.3 \%$ & 0.054 & 0.006 & $1.08(1.02-1.13)$ \\
\hline & Thyroid cancer & 4 & $33.2 \%$ & 0.213 & 0.012 & $1.27(1.05-1.53)$ \\
\hline & Bladder cancer & 3 & $87.1 \%$ & $P<0.001$ & 0.038 & $0.71(0.41-1.23)$ \\
\hline & Lung cancer & 3 & $26.7 \%$ & 0.255 & 0.132 & $0.87(0.73-1.04)$ \\
\hline & Others & 11 & $0.0 \%$ & 0.935 & 0.710 & $1.02(0.92-1.13)$ \\
\hline & \multicolumn{6}{|l|}{ Method } \\
\hline & PCR-RFLP & 12 & $0.0 \%$ & 0.981 & 0.590 & $1.03(0.93-1.14)$ \\
\hline & Sequencing & 2 & $0.0 \%$ & 0.946 & 0.001 & $4.00(1.79-8.94)$ \\
\hline & TaqMan & 13 & $57.1 \%$ & 0.006 & 0.696 & $1.02(0.92-1.14)$ \\
\hline & PCR & 4 & $72.9 \%$ & 0.011 & 0.780 & $1.05(0.76-1.44)$ \\
\hline & ND & 6 & $35.1 \%$ & 0.173 & 0.205 & $1.04(0.98-1.11)$ \\
\hline & Others & 3 & $0.0 \%$ & 0.577 & 0.089 & $1.25(0.97-1.63)$ \\
\hline & \multicolumn{6}{|l|}{ Source of control } \\
\hline & $\mathrm{HB}$ & 23 & $56.0 \%$ & 0.001 & 0.421 & 1.05 (0.93-1.18) \\
\hline & $\mathrm{pb}$ & 12 & $0.0 \%$ & 0.803 & 0.002 & $1.09(1.03-1.15)$ \\
\hline
\end{tabular}


Table 3 (continued)

\begin{tabular}{cllllll}
\hline Comparison & Subgroup & Number & $\mathbf{I}^{\mathbf{2}}$ & $\mathbf{P}_{\mathbf{H}}$ & $\mathbf{P}_{\mathbf{Z}}$ & $\mathbf{O R}(\mathbf{9 5} \% \mathbf{C l})$ \\
\hline & MIXED & 1 & NA & NA & 0.518 & $0.89(0.62-1.27)$ \\
& Nested & 4 & $0.0 \%$ & 0.989 & 0.160 & $0.91(0.80-1.04)$ \\
& Sample size & & & & \\
& $<100$ & 3 & $31.6 \%$ & 0.232 & 0.003 & $2.82(1.42-5.57)$ \\
& $>100$ & 37 & $32.9 \%$ & 0.029 & 0.153 & $1.04(0.99-1.10)$ \\
& Year & & & & 0.047 \\
& 2010 & 20 & $0.0 \%$ & 0.667 & $1.05(1.00-1.10)$ \\
& $>2010$ & 20 & $60.8 \%$ & 0.000 & 0.278 & $1.08(0.94-1.25)$ \\
\hline
\end{tabular}

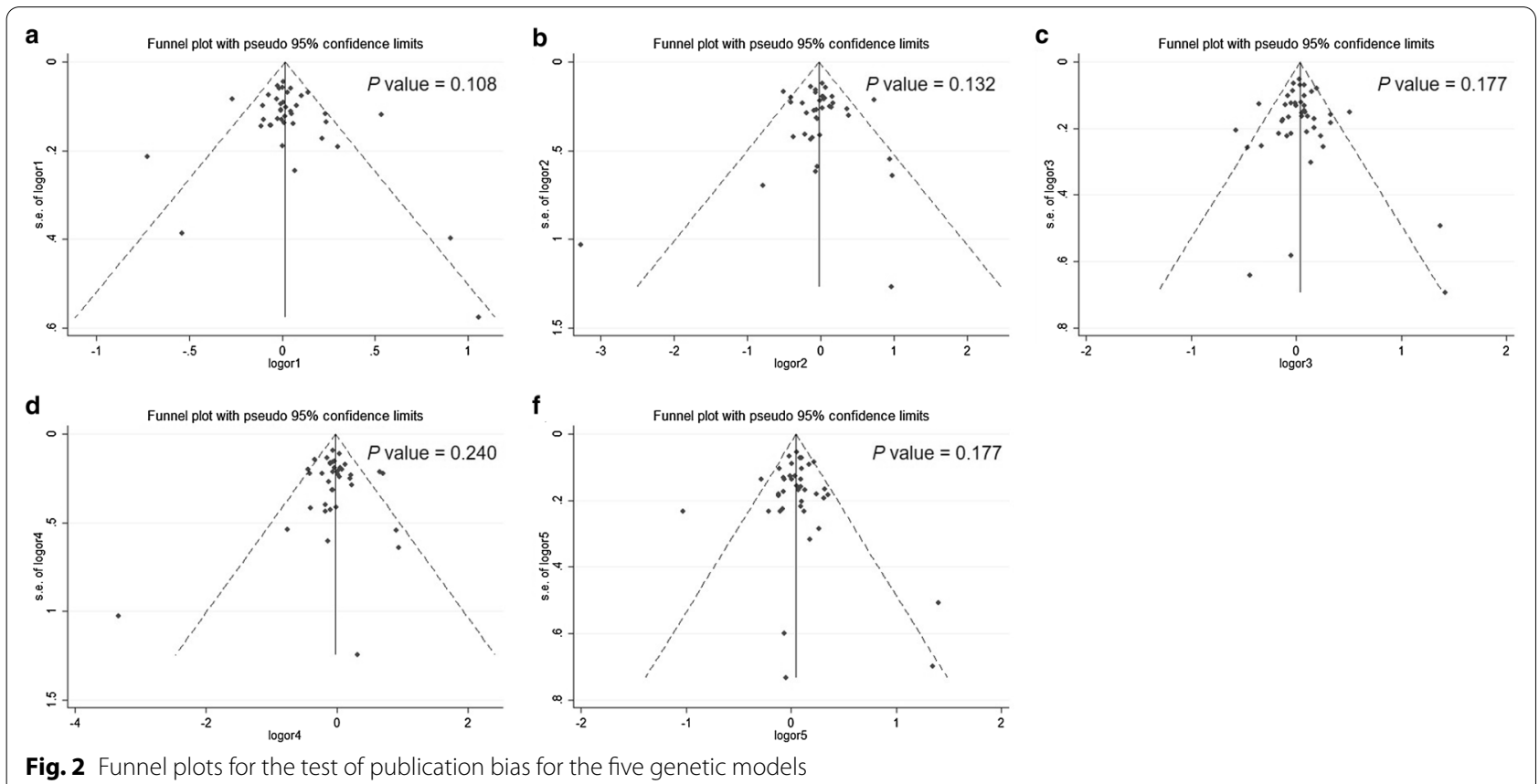

populations. In addition, detection method, source of control, and sample size played a role in heterogeneity and in the results. Well-designed large-scale studies are required to further evaluate the results.

\section{Abbreviations}

SNP: Single nucleotide polymorphism; XRCC3: X-ray repair cross-complementing group 3; HR: Homologous recombination; OR: Odds ratio; Cl: Confidence interval.

\section{Acknowledgements}

Not applicable

\section{Authors' contributions}

WQL: Drafting of manuscript/Analysis and interpretation of data. SMM, LL, ZYK, HBZ: Acquisition of data/Analysis and interpretation of data/Critical revision. JY: Study conception and design/Analysis and interpretation of data/Critical revision. All authors have read and approved the final manuscript.

\section{Funding}

This work was supported by the Applied Basic Foundation of Yunnan Province (No. 202001AT070009), Yunnan Health Training Project of High Level Talents (No. D-2019032). The funding bodies had no role in the research design, the conduct of the study, or the decision to publish the results.

\section{Availability of data and materials}

All data generated or analyzed during this study are included in this manuscript.

\section{Declarations}

Ethics approval and consent to participate

Not applicable.

Consent for publication

Not applicable. 


\section{Competing interests}

The authors declare that they have no competing interests.

\section{Author details}

${ }^{1}$ Department of Internal Medicine-Oncology, the First Affiliated Hospital of Kunming Medical University, Yunnan Province, Kunming 650032, People's Republic of China. ${ }^{2}$ Department of Oncology, the First Affiliated Hospital of Kunming Medical University, Yunnan Province, No. 295 Xichang Road, Kunming 650032, People's Republic of China.

Received: 14 February 2021 Accepted: 22 April 2021

Published online: 30 April 2021

\section{References}

1. Bray F, Ferlay J, Soerjomataram I, Siegel RL, Torre LA, Jemal A. Global cancer statistics 2018: GLOBOCAN estimates of incidence and mortality worldwide for 36 cancers in 185 countries. CA Cancer J Clin. 2018:68(6):394-424.

2. Roukos DH. Genome-wide association studies: how predictable is a person's cancer risk? Expert Rev Anticancer Ther. 2009;9(4):389-92.

3. Li Q, Guan R, Qiao Y, Liu C, He N, Zhang X, Jia X, Sun H, Yu J, Xu L. Association between the BRCA2 rs144848 polymorphism and cancer susceptibility: a meta-analysis. Oncotarget. 2017;8(24):39818-32.

4. Liu Y, Liu K, Zhao X, Sun Y, Ma N, Tang L, Yang H, Gao X, Yan L, Yuan M, et al. Impact of XPF rs2276466 polymorphism on cancer susceptibility: a meta-analysis. Biosci Rep. 2019;39(5).

5. Risch $N$, Merikangas $K$. The future of genetic studies of complex human diseases. Science (New York, NY). 1996;273(5281):1516-7.

6. Khanna KK, Jackson SP. DNA double-strand breaks: signaling, repair and the cancer connection. Nat Genet. 2001;27(3):247-54.

7. Ariyandy A, Sakai C, Ishida M, Mizuta R, Miyagawa K, Tashiro S, Kinomura A, Hiraaki K, Ueda K, Yoshizumi M, et al. XRCC3 polymorphism is associated with hypertension-induced left ventricular hypertrophy. Hypertens Res. 2018;41(6):426-34.

8. Mishra A, Saxena S, Kaushal A, Nagaraju G. RAD51C/XRCC3 facilitates mitochondrial DNA replication and maintains integrity of the mitochondrial genome. Mol Cell Biol. 2018;38(3).

9. Alkasaby MK, Abd El-Fattah Al, Ibrahim IH, Abd El-Samie HS. Polymorphism of XRCC3 in Egyptian breast cancer patients. Pharmgenomics Pers Med. 2020;13:273-82.

10. Auranen A, Song H, Waterfall C, Dicioccio RA, Kuschel B, Kjaer SK, Hogdall E, Hogdall C, Stratton J, Whittemore AS, et al. Polymorphisms in DNA repair genes and epithelial ovarian cancer risk. Int J Cancer. 2005;117(4):611-8.

11. Liu L, Miao L, Ji G, Qiang F, Liu Z, Fan Z. Association between XRCC1 and XRCC3 polymorphisms and colorectal cancer risk: a meta-analysis of 23 case-control studies. Mol Biol Rep. 2013;40(6):3943-52.

12. Shi CL, Li R, Xiong LW, Gu AQ, Han BH, Gu W. Lack of association between XRCC3 rs861539 (C>T) polymorphism and lung cancer risks: an update meta-analysis. Tumour Biol. 2013;34(3):1819-24.

13. Adel Fahmideh M, Schwartzbaum J, Frumento P, Feychting M. Association between DNA repair gene polymorphisms and risk of glioma: a systematic review and meta-analysis. Neuro Oncol. 2014;16(6):807-14.

14. Chen YT, Chen SY, Lin YJ, Huang CM, Chang YY, Tsai FJ. Association between XRCC3 Thr241Met SNP and systemic lupus erythematosus in Han Chinese patients in Taiwan, and a meta-analysis of healthy populations. J Clin Lab Anal. 2014;28(2):118-23.

15. Ma Q, Zhao Y, Wang S, Zhang X, Zhang J, Du M, Li L, Zhang Y. Genetic polymorphisms of XRCC3 Thr241Met (C18067T, rs861539) and bladder cancer risk: a meta-analysis of 18 research studies. Tumour Biol. 2014;35(2):1473-80

16. Yan Y, Liang H, LiT, Guo S, Li M, Qin X, Li S. Association of XRCC3 Thr241Met polymorphism and leukemia risk: evidence from a metaanalysis. Leuk Lymphoma. 2014;55(9):2130-4.

17. Zhang EJ, Cui ZG, Xu ZF, Duan WY, Huang SH, Tan XX, Yin ZH, Sun CF, Lu L. Lack of influence of an XRCC3 gene polymorphism on oral cancer susceptibility: meta-analysis. Asian Pac J Cancer Prev. 2014;15(23):10329-34.
18. Bei L, Xiao-Dong T, Yu-Fang G, Jian-Ping S, Zhao-Yu Y. DNA repair gene XRCC3 Thr241Met polymorphisms and lung cancer risk: a meta-analysis. Bull Cancer. 2015;102(4):332-9.

19. Cheng S, Wang L, Wang L, Wang Z. Association of XRCC3 gene rs861539 polymorphism with gastric cancer risk: evidence from a case-control study and a meta-analysis. Int J Clin Exp Pathol. 2015;8(2):1911-9.

20. Guo CX, Yang GP, Pei Q, Yin JY, Tan HY, Yuan H. DNA repair gene polymorphisms do not predict response to radiotherapy-based multimodality treatment of patients with rectal cancer: a meta-analysis. Asian Pac J Cancer Prev. 2015;16(2):713-8.

21. Cui Q, Zuo XY, Lian YF, Feng QS, Xia YF, He CY, Chen LZ, Jia WH, Mai HQ, Zeng YX, et al. Association between XRCC3 Thr241Met polymorphism and nasopharyngeal carcinoma risk: evidence from a large-scale casecontrol study and a meta-analysis. Tumour Biol. 2016;37(11):14825-30.

22. Qi L, Yu HQ, Zhang Y, Ding LJ, Zhao DH, Lv P, Wang WY, Xu Y. A Comprehensive meta-analysis of genetic associations between key polymorphic loci in DNA repair genes and Glioma risk. Mol Neurobiol. 2017;54(2):1314-25.

23. Sobhan MR, Forat Yazdi M, Mazaheri M, Zare Shehneh M, Neamatzadeh H. Association between the DNA repair gene XRCC3 rs861539 polymorphism and risk of osteosarcoma: a systematic review and meta-analysis. Asian Pac J Cancer Prev. 2017;18(2):549-55.

24. Tan LM, Qiu CF, Zhu T, Jin YX, Li X, Yin JY, Zhang W, Zhou HH, Liu ZQ. Genetic polymorphisms and platinum-based chemotherapy treatment outcomes in patients with non-small cell lung cancer: a genetic epidemiology study based meta-analysis. Sci Rep. 2017;7(1):5593.

25. Yu SN, Liu GF, Li XF, Fu BH, Dong LX, Zhang SH. Evaluation of prediction of polymorphisms of DNA repair genes on the efficacy of platinum-based chemotherapy in patients with non-small cell lung cancer: a network meta-analysis. J Cell Biochem. 2017;118(12):4782-91.

26. Wang $X$, Liu Z. Systematic meta-analysis of genetic variants associated with osteosarcoma susceptibility. Medicine. 2018;97(38):e12525.

27. Zhao Y, Zhao E, Zhang J, Chen Y, Ma J, Li H. A comprehensive evaluation of the association between polymorphisms in XRCC1, ERCC2, and XRCC3 and prognosis in hepatocellular carcinoma: a meta-analysis. J Oncol. 2019:2019:2408946.

28. Han S, Zhang HT, Wang Z, Xie Y, Tang R, Mao Y, Li Y. DNA repair gene XRCC3 polymorphisms and cancer risk: a meta-analysis of 48 case-control studies. European journal of human genetics : EJHG. 2006;14(10):1136-44.

29. Dashti S, Taherian-Esfahani Z, Keshtkar A, Ghafouri-Fard S. Associations between XRCC3 Thr241Met polymorphisms and breast cancer risk: systematic-review and meta-analysis of 55 case-control studies. BMC Med Genet. 2019;20(1):79.

30. He XF, Wei W, Su J, Yang ZX, Liu Y, Zhang Y, Ding DP, Wang W. Association between the XRCC3 polymorphisms and breast cancer risk: meta-analysis based on case-control studies. Mol Biol Rep. 2012;39(5):5125-34.

31. Yuan C, Liu X, Yan S, Wang C, Kong B. Analyzing association of the XRCC3 gene polymorphism with ovarian cancer risk. Biomed Res Int. 2014:2014:648137.

32. Chai F, Liang Y, Chen L, Zhang F, Jiang J. Association between XRCC3 Thr241Met polymorphism and risk of breast cancer: meta-analysis of 23 case-control studies. Med Sci Monit. 2015;21:3231-40.

33. Peng Q, Mo C, Tang W, Chen Z, Li R, Zhai L, Yang S, Wu J, Sui J, Li S. DNA repair gene XRCC3 polymorphisms and bladder cancer risk: a metaanalysis. Tumor Biol. 2014;35(3):1933-44.

34. Qiu LX, Mao C, Yao L, Yu KD, Zhan P, Chen B, Liu HG, Yuan H, Zhang J, Xue K, et al. XRCC3 5'-UTR and IVS5-14 polymorphisms and breast cancer susceptibility: a meta-analysis. Breast Cancer Res Treat. 2010;122(2):489-93.

35. Andreas S. Critical evaluation of the Newcastle-Ottawa scale for the assessment of the quality of nonrandomized studies in meta-analyses. Eur J Epidemiol. 2010;25(9):603-5.

36. Attia J, Thakkinstian A, D'Este C. Meta-analyses of molecular association studies: methodologic lessons for genetic epidemiology. J Clin Epidemiol. 2003:56(4):297-303

37. Lau J, loannidis JP, Schmid CH. Quantitative synthesis in systematic reviews. Ann Intern Med. 1997;127(9):820-6.

38. Higgins JP, Thompson SG, Deeks JJ, Altman DG. Measuring inconsistency in meta-analyses. BMJ. 2003;327(7414):557-60.

39. DerSimonian R, Laird N. Meta-analysis in clinical trials. Control Clin Trials. 1986;7(3):177-88. 
40. Mackawy AMH, Shalaby SM, Megahed OY. DNA repair gene (XRCC3) polymorphisms and its association with ovarian carcinoma in Egyptian PATIENTS. Meta Gene. 2019;21.

41. Pei JS, Chang WS, Hsu PC, Chen CC, Cheng SP, Wang YC, Tsai CW, Shen TC, Bau DT. The contribution of XRCC3 genotypes to childhood acute lymphoblastic leukemia. Cancer Manage Res. 2018;10:5677-84.

42. Sarwar R, Mahjabeen I, Bashir K, Saeed S, Kayani MA. Haplotype based analysis of XRCC3 gene polymorphisms in thyroid cancer. Cell Physiol Biochem. 2017:42(1):22-33.

43. De Mattia E, Cecchin E, Polesel J, Bignucolo A, Roncato R, Lupo F, Crovatto M, Buonadonna A, Tiribelli C, Toffoli G. Genetic biomarkers for hepatocellular cancer risk in a caucasian population. World J Gastroenterol. 2017;23(36):6674-84.

44. Al Zoubi MS, Zavaglia K, Mazanti C, Al Hamad M, Al Batayneh K, Aljabali AAA, Bevilacqua G. Polymorphisms and mutations in GSTP1, RAD51, XRCC1 and XRCC3 genes in breast cancer patients. Int J Biol Markers. 2017;32(3):e337-43.

45. Zhu G, Su H, Lu L, Guo H, Chen Z, Sun Z, Song R, Wang X, Li H, Wang Z. Association of nineteen polymorphisms from seven DNA repair genes and the risk for bladder cancer in gansu province of China. Oncotarget. 2016;7(21):31372-83.

46. Yuan $K$, Huo M, Sun Y, Wu H, Chen H, Wang Y, Fu R. Association between $x$-ray repair cross-complementing group 3 (XRCC3) genetic polymorphisms and papillary thyroid cancer susceptibility in a Chinese Han population. Tumor Biol. 2016;37(1):979-87.

47. Yan L, Li Q, Li X, Ji H, Zhang L. Association studies between XRCC1, XRCC2, XRCC3 polymorphisms and differentiated thyroid carcinoma. Cell Physiol Biochem. 2016;38(3):1075-84.

48. Ali AM, Abdulkareem H, Al Anazi M, Reddy Parine N, Shaik JP, Alamri A, Ali Khan Pathan A, Warsy A. Polymorphisms in DNA repair gene XRCC3 and susceptibility to breast cancer in Saudi females. BioMed Res Int. 2016;2016.

49. Su CH, Chang WS, Hu PS, Hsiao CL, Ji HX, Liao CH, Yueh TC, Chuang CL, Tsai CW, Hsu CM, et al. Contribution of DNA double-strand break repair gene XRCC3 genotypes to triple-negative breast cancer risk. Cancer Genomics Proteomics. 2015;12(6):359-68.

50. Liu JC, Tsai CW, Hsu CM, Chang WS, Li CY, Liu SP, Shen WC, Bau DT. Contribution of double strand break repair gene XRCC3 genotypes to nasopharyngeal carcinoma risk in Taiwan. Chin J Physiol. 2015; 58(1).

51. Goricar K, Kovac V, Jazbec J, Lamovec J, Dolzan V. Homologous recombination repair polymorphisms and the risk for osteosarcoma. J Med Biochem. 2015;34(2):200-6.

52. Goričar K, Erčulj N, Faganel Kotnik B, Debeljak M, HovnikT, Jazbec J, Dolžan $\mathrm{V}$. The association of folate pathway and DNA repair polymorphisms with susceptibility to childhood acute lymphoblastic leukemia. Gene. 2015:562(2):203-9.

53. Chen HJ, Chang WS, Hsia TC, Miao CE, Chen WC, Liang SJ, Chen AC, Chang JG, Tsai CW, Hsu CM, et al. Contribution of genotype of DNA double-strand break repair gene XRCC3, gender, and smoking behavior to lung cancer risk in Taiwan. Anticancer Res. 2015;35(7):3893-900.

54. Chang WS, Tsai CW, Wang JY, Ying TH, Hsiao TS, Chuang CL, Yueh TC, Liao CH, Hsu CM, Liu SP, et al. Contribution of $x$-ray repair complementing defective repair in Chinese hamster cells 3 (XRCC3) genotype to leiomyoma risk. Anticancer Res. 2015;35(9):4691-6.

55. Al Zoubi MS. X-ray repair cross-complementing protein 1 and 3 polymorphisms and susceptibility of breast cancer in a Jordanian population. Saudi Med J. 2015;36(10):1163-7.

56. Tsai CW, Chang WS, Liu JC, Tsai MH, Lin CC, Bau DT. Contribution of DNA double-strand break repair gene XRCC3 genotypes to oral cancer susceptibility in Taiwan. Anticancer Res. 2014;34(6):2951-6.

57. Smolkova B, Dusinska M, Hemminki K. NBN and XRCC3 genetic variants in childhood acute lymphoblastic leukaemia. Cancer Epidemiol. 2014.

58. Zhao P, Zou P, Zhao L, Yan W, Kang C, Jiang T, You Y. Genetic polymorphisms of DNA double-strand break repair pathway genes and glioma susceptibility. BMC Cancer. 2013;13.
59. He F, Chang SC, Wallar GM, Zhang ZF, Cai L. Association of XRCC3 and XRCC4 gene polymorphisms, family history of cancer and tobacco smoking with non-small-cell lung cancer in a Chinese population: a casecontrol study. J Hum Genet. 2013;58(10):679-85.

60. Gresner P, Gromadzinska J, Polanska K, Twardowska E, Jurewicz J, Wasowicz W. Genetic variability of Xrcc3 and Rad51 modulates the risk of head and neck cancer. Gene. 2012;504(2):166-74.

61. Vral A, Willems P, Claes K, Poppe B, Baeyens A, Perletti G, Thierens H. Combined effect of polymorphisms in Rad51 and XRCC3 on breast cancer risk and chromosomal radiosensitivity. Mol Med Rep. 2011;4(5):901-12.

62. Quaye L, Tyrer J, Ramus SJ, Song H, Wozniak E, DiCioccio RA, McGuire V, Hogdall E, Hogdall C, Blaakaer J, et al. Association between common germline genetic variation in 94 candidate genes or regions and risks of invasive epithelial ovarian cancer. PLoS ONE. 2009;4(6):e5983.

63. Andrew AS, Mason RA, Kelsey KT, Schned AR, Marsit CJ, Nelson HH, Karagas MR. DNA repair genotype interacts with arsenic exposure to increase bladder cancer risk. Toxicol Lett. 2009;187(1):10-4.

64. Hayden PJ, Tewari P, Morris DW, Staines A, Crowley D, Nieters A, Becker N, Desanjosé $S$, Foretova $L$, Maynadié $M$, et al. Variation in DNA repair genes XRCC3, XRCC4, XRCC5 and susceptibility to myeloma. Hum Mol Genet. 2007; 16(24):3117-27.

65. Wu X, Gu J, Grossman HB, Amos Cl, Etzel C, Huang M, Zhang Q, Millikan $R E$, Lerner S, Dinney CP, et al. Bladder cancer predisposition: a multigenic approach to DNA-repair and cell-cycle-control genes. Am J Hum Genet. 2006;78(3):464-79.

66. Pharoah P. Commonly studied single-nucleotide polymorphisms and breast cancer: results from the Breast Cancer Association Consortium. J Natl Cancer Inst. 2006;98(19):1382-96.

67. Ni HX, Bian JC, Shen Q, Tang HW, Zhu QX, Wu Y. Genetic polymorphisms of XRCC 3 and susceptibility of papillary thyroid carcinoma. Fudan Univ J Med Sci. 2006;33(2):147-52.

68. García-Closas M, Egan KM, Newcomb PA, Brinton LA, Titus-Ernstoff L, Chanock S, Welch R, Lissowska J, Peplonska B, Szeszenia-Dabrowska N, et al. Polymorphisms in DNA double-strand break repair genes and risk of breast cancer: Two population-based studies in USA and Poland, and meta-analyses. Hum Genet. 2006;119(4):376-88.

69. Matullo G, Guarrera S, Sacerdote C, Polidoro S, Davico L, Gamberini S, Karagas M, Casetta G, Rolle L, Piazza A, et al. Polymorphisms/haplotypes in DNA repair genes and smoking: A bladder cancer case-control study. Cancer Epidemiol Biomarkers Prev. 2005;14(11 I):2569-78.

70. Tranah GJ, Giovannucci E, Ma J, Fuchs C, Hankinson SE, Hunter DJ. XRCC2 and XRCC3, polymorphisms are not associated with risk of colorectal adenoma. Cancer Epidemiol Biomarkers Prev. 2004;13(6):1090-1.

71. Jacobsen NR, Raaschou-Nielsen O, Nexø B, Wallin H, Overvad K, Tjønneland A, Vogel U. XRCC3 polymorphisms and risk of lung cancer. Cancer Lett. 2004;213(1):67-72.

72. Han J, Hankinson SE, Ranu H, De Vivo I, Hunter DJ. Polymorphisms in DNA double-strand break repair genes and breast cancer risk in the Nurses' Health Study. Carcinogenesis. 2004;25(2):189-95.

73. Han J, Hankinson SE, Hunter DJ, De Vivo I. Genetic variations in XRCC2 and XRCC3 are not associated with endometrial cancer risk. Cancer Epidemiol Biomarkers Prev. 2004;13(2):330-1.

74. Kuschel B, Auranen A, McBride S, Novik KL, Antoniou A, Lipscombe JM, Day NE, Easton DF, Ponder BAJ, Pharoah PDP, et al. Variants in DNA double-strand break repair genes and breast cancer susceptibility. Hum Mol Genet. 2002;11(12):1399-407.

75. Winsey SL, Haldar NA, Marsh HP, Bunce M, Marshall SE, Harris AL, Wojnarowska F, Welsh Kl. A variant within the DNA repair gene XRCC3 is associated with the development of melanoma skin cancer. Cancer Res. 2000:60(20):5612-6.

\section{Publisher's Note}

Springer Nature remains neutral with regard to jurisdictional claims in published maps and institutional affiliations. 